\title{
Multicarrier CDMA with Adaptive Frequency Hopping for Mobile Radio Systems
}

\author{
Qingxin Chen, Elvino S. Sousa, Member, IEEE, \\ and Subbarayan Pasupathy, Fellow, IEEE
}

\begin{abstract}
A modified multicarrier (MC) direct-sequence codedivision multiple-access (DS-CDMA) system has been proposed for use over slow multipath fading channels with frequency selectivity in the reverse link transmission of a cellular network. Instead of transmitting data substreams uniformly through subchannels, data substreams hop over subchannels with the hopping patterns adaptively adjusted to the channel fading characteristics. The problem of determining the optimal hopping pattern is formulated as a multiobjective optimization problem, for which an efficient algorithm, based on the water-filling (WF) principle, is designed to solve the problem practically. Simulation results show that the performance in terms of the average bit-error probability (BEP) (over all users) is better than that of single carrier RAKE receiver systems, conventional MC CDMA systems applying moderate error protection, or diversity systems with different combining techniques.
\end{abstract}

\section{INTRODUCTION}

$\mathbf{V}$ ARIOUS multicarrier (MC) transmission schemes have been recently introduced into direct-sequence codedivision multiple-access (DS-CDMA) systems to acquire benefits such as higher rate data transmission, bandwidth efficiency, frequency diversity, and interference reduction. Based on the methods by which MC modulation and sequence spreading are implemented, these schemes can be classified under three categories as proposed in [1]-[8].

In this paper, we are interested in the improvement of a $\mathrm{MC}$ scheme where the total system bandwidth $W$ is divided into $M$ equiwidth subchannels, and the serial data stream of each user (mobile unit) is converted into $M$ parallel substreams with each being transmitted as a narrow band DS signal over one of the subchannels [9]. This scheme bears close resemblance to the one proposed by Kondo et al. [5]-[7] except that it eliminates the frequency diversity transmission (equivalent to a rate $1 / M$ repetition code) which may reduce the spectral efficiency of the system. In a channel affected only by additive white Gaussian noise (AWGN), the system in [9] is simple to implement, and with a longer symbol time, it offers the advantages of easier sequence synchronization and multipleaccess interference (MAI) reduction, which in turn may lead to an increase in performance over a single carrier DSCDMA system (SC-DS-CDMA). However, in the presence of multipath fading, the MC system is disadvantageous when compared to a SC-DS-CDMA system using a RAKE receiver.

Manuscript received May 29, 1995; revised October 10, 1995.

The authors are with the Department of Electrical and Computer Engineering, University of Toronto, Canada (email: sousa@comm.toronto.edu).

Publisher Item Identifier S 0733-8716(96)05237-7.
This is because the resolvability of the different paths in a given subchannel is reduced, hence, the effect of path diversity is reduced. With time-varying flat fading over the subchannels, data substreams transmitted over severely deteriorated subchannels may incur a long burst of errors. Error control coding with interleaving has been suggested to recover such performance losses [10]; however, this approach may have disadvantages in terms of spectral efficiency, implementation complexity, and transmission delay.

For multipath fading channels, we propose the following modifications to the above MC scheme: 1) Rather than transmitting one data substream over each subchannel, data substreams can hop to any of the subchannels depending on the state of the fading process in a subchannel; 2) since it is possible for more than one substream belonging to the same user to hop to the same subchannel, $M$ (the number of carriers) pseudo-random noise (PN) sequences have to be assigned to each user so that every data substream can be uniquely identified.

The proposed system may appear similar to a conventional hybrid frequency-hopping/direct-sequence (FH/DS) CDMA system in the sense that a data substream can hop to any one of the subchannels, where a narrow-band DS waveform is in use [11]. However, these two schemes differ as follows: First, rather than transmitting over one particular subchannel during any hopping interval as is done in the conventional FH/DS system, the signal is split into substreams and transmitted over a few chosen subchannels simultaneously. While every substream must have constant rate, users can use a different number of substreams up to a maximum of $M$, which offers the potential to provide multirate services without any increase in the system complexity. The second difference is that instead of random or deterministic hopping patterns as adopted in conventional FH systems, the hopping pattern in the proposed system is adaptively determined by the channel fading process.

By exploiting the channel-state information, the modified MC system outperforms the conventional SC-DS-CDMA system with RAKE reception without incurring any bandwidth expansion associated with coding or diversity schemes. It is especially suitable for operating in channels with very slow time variations which are often encountered by slow moving vehicles or pedestrians.

The system model is described in Section II. In Section III the problem of obtaining the optimal hopping pattern is formulated, and an algorithm is devised to solve the optimization problem efficiently. Numerical results from simulation are 


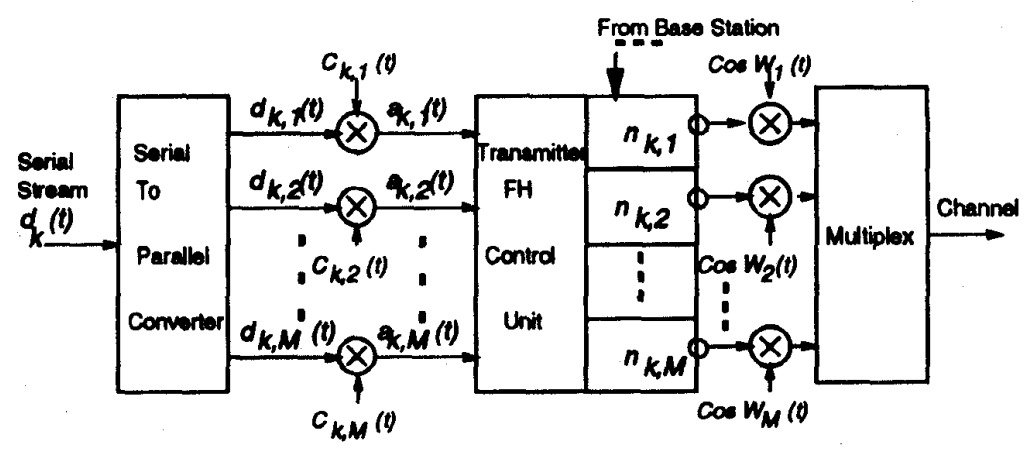

(a)

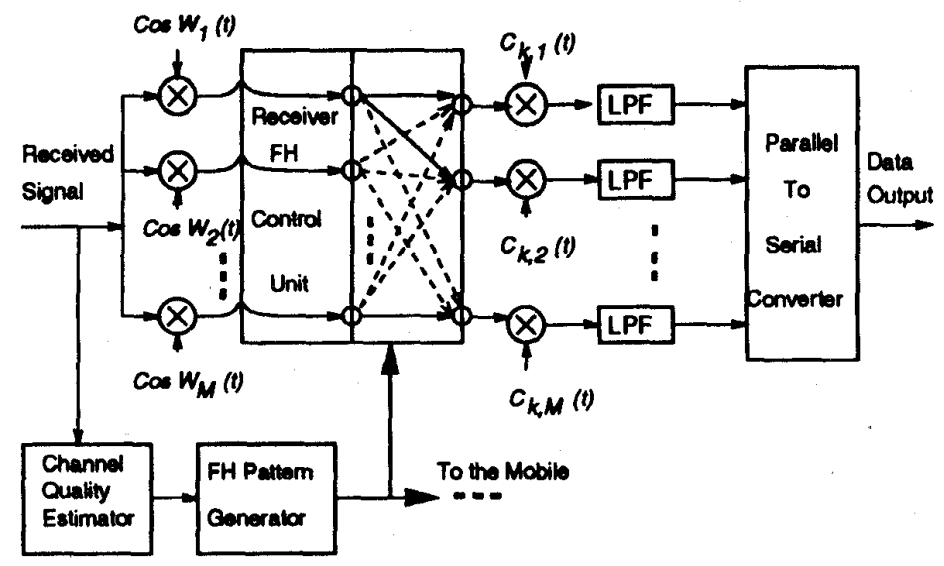

(b)

Fig. 1. A MC DS-CDMA system with adaptive frequency hopping for the k:th user: (a) transmitter and (b) receiver.

shown and a comparison is made with other systems in Section IV. Section V concludes the paper.

\section{SYSTEM MODELS}

We consider the reverse link of a CDMA cellular system. An adaptive MC FH/DS transmission scheme for the $k$ th user of this system is shown in Fig. 1.

\section{A. Transmitter Model}

At the transmitter, the user's binary data stream $d_{k}(t)$ of rate $R$ is converted into $M$ interleaved substreams $D_{k}(t)=$ $\left(d_{k, 1}(t), d_{k, 2}(t), \cdots, d_{k, M}(t)\right)$. The $m$ th substream consists of bits $m, M+m, 2 M+m, \cdots$. The data substreams are transmitted over subchannels with a reduced rate $\frac{R}{M}$, and the data interval over subchannels is $T=\frac{M}{R}$. The substreams are spread by random sequences $C_{k}(t)=\left(c_{k, 1}(t), c_{k, 2}(t), \cdots, c_{k, M}(t)\right)$, respectively, resulting in another vector of direct-sequence signals $A_{k}(t)=\left(a_{k, 1}(t), a_{k, 2}(t), \cdots, a_{k, M}(t)\right)$. Although orthogonal spreading sequences would be preferable for the purpose of reducing MAI, due to the adaptive frequency hopping all substreams from all users may hop to the same subband. For a system with $K$ users and $M$ subchannels, this would require a maximum of $N_{s}=K M$ orthogonal sequences. However, the maximum number of orthogonal sequences in a given subchannel is $N=W / R . N_{s}$ is normally much larger than $N$ in a practical system.
Assuming binary phase shift keying (BPSK) modulation, each $a_{k, i}(t) \in A_{k}(t)$ is to modulate one of the carrier frequencies in $\Omega=\left(\omega_{1}, \omega_{2}, \cdots, \omega_{M}\right)$. Carrier frequencies are equally separated by the chip rate $\frac{1}{T_{c}}$, and the corresponding signals are mutually orthogonal in the interval $[0, T]$. As has been said, the $k$ th user's $m$ th substream $d_{k, m}(t)$ is not necessarily transmitted over the $m$ th subchannel. Letting $L_{k}=\left(l_{k, 1}, l_{k, 2}, \cdots, l_{k, M}\right)$ denote the set of subchannels which are actually occupied by $D_{k}(t)$ during one frequency hopping period of duration $T_{f h}, L_{k}$ indicates the mapping between $D_{k}(t)$ and $\Omega$, i.e., $\omega_{l_{k, m}} \in \Omega$ is the carrier frequency modulated by $a_{k, m}(t)$. Note that since more than one of each user's substreams may hop to the same subchannel, two or more elements in $L_{k}$ may have the same value. Without loss of generality we assume $\omega_{l_{k, 1}} \leq \omega_{l_{k, 2}}, \cdots \leq \omega_{l_{k, M}}$, i.e., substreams enter the the subchannels in the natural order. The mapping vector $L_{k}$ is adaptively determined by the transmitter FH control unit (TFHCU) upon receiving instructions from the base station through a feedback control channel.

The transmitted signal from the $k$ th user is written as

$$
\begin{aligned}
s_{k}(t)= & \sum_{m=1}^{M} \sum_{i=-\infty}^{\infty} \sqrt{2 P} d_{k, m}(t-\lfloor i / N\rfloor T) \\
& \times c_{k, m}\left(t-i T_{c}\right) p\left(t-i T_{c}\right) \cos \omega_{l_{k, m}} t \\
= & \sum_{m=1}^{M} \sqrt{2 P} \hat{a}_{k, m}(t) \cos \omega_{l_{k, m}} t
\end{aligned}
$$


where $P$ is the transmitted power per substream, $p(t)$ is the chip pulse shape, and $\hat{a}_{k, m}(t)$ is the data modulated PN pulse train. $\lfloor x\rfloor$ denotes the largest integer less than $x$.

To avoid the issue of intersubchannel interference, which is not the focus here, a frequency limited sinc chip pulse is used, i.e., $p(t)$ in (1) is

$$
p(t)=\frac{\sin \frac{\pi t}{T_{c}}}{\frac{\pi t}{T_{c}}}
$$

\section{B. Channel Model}

The transmission bandwidth available for a CDMA system is normally much larger than the data rate. The entire frequency band is generally modeled as a dispersive Rayleighfading channel with time variation and frequency selectivity. We model the $k$ th user's entire channel as a linear filter with the following low-pass equivalent complex-valued impulse response [12]

$$
h_{k}(t)=\sum_{l=0}^{L_{p}} \rho_{k, l} \delta\left(t-\tau_{k, l}\right) \exp \left(j \varphi_{k, l}\right)
$$

where $L_{p}$ is the number of paths, $\rho_{k, l}, \tau_{k, l}$, and $\varphi_{k, l}$ are the path amplitude, time delay, and signal phase in the $l$ th path, respectively. The fading amplitudes $\alpha_{k, l}$ are modeled as independent Rayleigh random variables (RV's) with unit second moment. $\phi_{k, l}$ and $\tau_{k}$ are independent RV's uniformly distributed in $[0,2 \pi)$ and $\left[0, T_{d}\right]$, respectively. The range of path delays $T_{d}$ is defined as the channel delay spread. All the fading parameters are assumed constant over at least one hopping period.

In our system, however, information is transmitted as narrow-band DS waveforms over subchannels whose bandwidth is a portion of the entire channel bandwidth. The subchannel bandwidth is

$$
W_{s}=\frac{1}{T_{c}}=\frac{W}{M} .
$$

A number of factors have to be considered in selecting the appropriate number of subchannels $M$. On one hand, for achieving quasisynchronization so as to reduce MAI, a larger $M$ is preferred so that the delay spread $T_{d}$ is a fraction of $T_{c}$. On the other hand, assuming that the hop interval is equal to the coherence time, a larger $M$ and thus a larger $T\left(=\frac{M}{R}\right)$ means that there are fewer data symbols transmitted during each hop, herice, a greater rate of adaptation. As a compromise between these two effects, we choose $M$ such that $T_{c} \approx T_{d}$. In doing so, $W_{s}$ is almost equal to the coherence bandwidth of the multipath channel, and thus the correlation between the fading amplitudes of adjacent subchannels should be less than 0.5 .

We derive the subchannel impulse response for the $k$ th user's $m$ th subchannel (referred to as the $(k, m)$ subchannel hereafter) by applying (3) to $M$ band-pass filters with each centered at one of the $M$ carrier frequencies and having a bandwidth of $W_{s}$ [10]. Let $H_{k}(\omega)$ be the Fourier transform of $h_{k}(t)$. The transfer function for the $(k, m)$ subchannel is

$$
H_{k, m}(\omega)=H_{k}(\omega) \Pi_{m}(\omega)
$$

where $\Pi_{m}(\omega)$ is the transfer function of an ideal bandpass filter with bandwidth $2 \pi W_{s}$ and center frequency $\omega_{m}$.

We write the subchannel low-pass equivalent impulse response as

$$
h_{k, m}(t)=\alpha_{k, m} \delta\left(t-\tau_{k}\right) \exp \left(j \phi_{k, m}\right)
$$

where $\alpha_{k, m}$ and $\phi_{k, m}$ are the fading amplitude and signal phase for the $(k, m)$ subchannel, respectively, and $\tau_{k}$ is the path delay. The parameters $\alpha_{k, m}$ and $\phi_{k, m}$ are the average of the amplitude and phase responses of the subchannel transfer function over the subchannel bandwidth. The path delay $\tau_{k}$ is obtained as the average of the multipath delays in (3), $\tau_{k}=\frac{1}{L_{p}} \sum_{l=0}^{l=L_{p}} \tau_{k, l}$.

\section{Receiver Model}

A conventional correlator detection is assumed at the receiver. The channel quality estimator (CQE) estimates the fading-channel parameters periodically and passes the estimates to the FH pattern generator (FHPG). Based on the channel parameters, the FHPG generates a $K \times M$ matrix $H$

$$
H=\left[\begin{array}{cccc}
n_{1,1} & n_{1,2} & \cdots & n_{1, M} \\
n_{2,1} & n_{2,2} & \cdots & n_{2, M} \\
\cdots & \cdots & \cdots & \cdots \\
n_{K, 1} & n_{K, 2} & \cdots & n_{K, M}
\end{array}\right]
$$

where $n_{k, m} \in[0, M]$ denotes the number of substreams hopping to the $(k, m)$ subchannel. Note that the sum of each row of $H$ equals $M$.

The kth row of $H$ is sent to the TFHCU through a feedback control channel, based on which the carrier modulation is carried out in the transmitter. The same information is also passed to the $k$ th user's receiver FH control unit (RFHCU) for completing the despreading process, accordingly. As shown in Fig. 1, the RFHCU functions like an $M \times M$ switch connecting the demodulated signals to their corresponding despreading sequences. The solid lines in the RFHCU in Fig. 1 are examples of actual connections during one hop period, whereas the dashed lines represent all the possible connections.

Applying (6) to (1), the received signal is

$$
\begin{aligned}
r(t)= & \sum_{k=1}^{K} \sum_{m=1}^{M} \sqrt{2 P} \alpha_{l_{k, m}} \hat{a}_{k, m}\left(t-\tau_{k}\right) \\
& \times \cos \left(\omega_{l_{k, m}} t+\phi_{l_{k, m}}\right)+n(t)
\end{aligned}
$$

where $n(t)$ is the AWGN with two-sided power spectral density $N_{0} / 2$.

It is seen that the success of the proposed system depends on the availability of a CQE providing reasonably accurate and timely estimation of the channel, a reliable feedback control channel, and a FHPG that promptly produces optimal frequency hopping patterns. The issue of fading-channel estimation has been intensively studied in general [13]-[15]. We assume that a reliable feedback control channel is available. In the rest of this paper, we focus on the algorithm design for the FHPG and on the performance analysis of the system. 


\section{ANALYSIS AND ALGORITHM DESIGN}

In the reverse link transmission, different users have different channels. These channels are statistically independent for two users separated geographically by a few wavelengths, which is the case in practice. The idea behind our scheme is to take advantage of this diversity among users as well as the frequency selectivity in each channel. With coherent detection, the parameter which measures the channel quality is the fading amplitude $\alpha_{k, m}$. Subchannels with $\alpha_{k, m}>1$, due to constructive interference, can enhance the signal levels, and those having $\alpha_{k, m}<1$ weaken the signal. Although it is seemingly desirable for one particular user to concentrate all its data substreams on the subchannel with the largest signal level, the user could be contributing a larger amount of interference and thus be harmful for other users who happen to be transmitting over the same subchannel. The key issue is to find the best way for positioning a user's substreams so that the lowest bit-error probability (BEP) can be achieved for all users.

\section{A. Analysis}

We are concerned with very slow fading channels. By "very slow fading channels," we imply $T_{f h} \gg T$. Without loss of generality, we detect the $k$ th user's $m$ th data substream $d_{k, m}(t)$ transmitted over the $l_{k, m}$ th subchannel.

Assuming bit " 1 " is transmitted, the low-pass filter (LPF) output is

$$
y_{k, m}=\frac{\gamma_{k, l_{k, m}} T}{2}+I_{k, m}+\eta_{k, m}
$$

where $\gamma_{k, m}=\alpha_{k, m} \sqrt{2 P}(8), I_{k, m}$ is the interference term, and $\eta_{k, m}$ is the noise term, which is a Gaussian RV with zero mean and variance $N_{0} T / 4$.

Considering a $[0, T]$ integrator as the LPF, $I_{k, m}$ can be expressed as

$$
\begin{aligned}
I_{k, m}= & \sum_{i=1}^{K} \frac{\gamma_{i, l_{k, m}}}{2} \cos \theta_{i, l_{k, m}} \\
& \times \int_{0}^{T} \sum_{\substack{j \\
l_{i, j}=l_{k, m} \& \\
(i, j) \neq(k, m)}} \hat{a}_{i, j}\left(t-\tau_{i}\right) \hat{c}_{k, m}(t) d t
\end{aligned}
$$

where $\hat{c}_{k, m}(t)=\sum_{n=-\infty}^{\infty} c_{k, m}\left(t-n T_{c}\right) p\left(t-n T_{c}\right)$, and $\theta_{i, l_{k, m}}=\phi_{i, l_{k, m}}-\phi_{k, l_{k, m}}$.

It has been shown that for systems with large $K$ and moderate to large $N$, a Gaussian distribution with variable variance is a good model for the MAI [16]. The average variance of $I_{k, m}$ is

$$
\begin{aligned}
V\left(I_{k, m}\right)= & \frac{N T_{d}^{2 .}}{8} \\
& \times\left[\sum_{\substack{i=1 \\
i \neq k}}^{K} n_{i, l_{k, m}} \gamma_{i, l_{k, m}}^{2}+\left(n_{k, l_{k, m}}-1\right) \gamma_{k, l_{k, m}}^{2}\right]
\end{aligned}
$$

The first term of (11) accounts for the interference from other users hopping to the same subchannel, and the second term accounts for interference from other substreams of the same user.

Conditioned on the channel parameters, the average BEP for transmitting $d_{k, m}(t)$ during one hop period $T_{f h}$ is $\mathbf{P}\left[y_{k, m}>0\right]$ which is given by

$$
p_{k, m}=\frac{1}{2} \operatorname{erfc} \sqrt{\lambda_{k, m}}
$$

where $\lambda_{k, m}$ is the signal-to-interference-plus-noise ratio (SINR)

$$
=\frac{\lambda_{k, m}}{N_{0}+\frac{\bar{T}_{d}^{2}}{N}\left[\sum_{\substack{i=1, l_{k, m} \\ i \neq k}}^{K} n_{i, l_{k, m}} v_{i, l_{k, m}}+\left(n_{k, l_{k, m}}-1\right) v_{k, l_{k, m}}\right]}
$$

where $\bar{T}_{d}=T_{d} / T_{c}$ is the normalized delay spread and $v_{k, m}=\frac{1}{2} \gamma_{k, m}^{2} T$ is the received energy per bit for the $(k, m)$ subchannel for the given hop. With open loop power control, the mean value of $v_{k, m}$ is a constant $E_{b}$.

The probability $p_{k, m}$ is a function of both the hopping pattern matrix $H$ and the received bit energy matrix $\Upsilon$ that can be obtained from the estimated fading amplitudes

$$
\Upsilon=\left[\begin{array}{cccc}
v_{1,1} & v_{1,2} & \cdots & v_{1, M} \\
v_{2,1} & v_{2,2} & \cdots & v_{2, M} \\
\cdots & \cdots & \cdots & \cdots \\
v_{K, 1} & v_{K, 2} & \cdots & v_{K, M}
\end{array}\right]
$$

The average BEP of the $k$ th user is, therefore

$$
\bar{p}_{k}=\frac{1}{M} \sum_{m=1}^{M} p_{k, m} .
$$

Let $P_{e}$ be the vector $\left(\bar{p}_{1}, \bar{p}_{2}, \cdots, \bar{p}_{K}\right)$. We use the average BEP among all users as the measure of system performance. To decrease the system BEP, minimizing each element of $P_{e}$ should be equally important. This leaves us with a multiobjective optimization problem formulated as follows:

$$
\begin{aligned}
& \min _{H \in \mathcal{H}} P_{e}=\left(\bar{p}_{1}, \bar{p}_{2}, \cdots, \bar{p}_{K}\right), \quad \forall \Upsilon \\
& \text { subject to: } \sum_{m=1}^{M} n_{k, m}=M, \quad \forall k
\end{aligned}
$$

where $\mathcal{H}$ is the set of matrices of the form in (7).

In general, a multiobjective optimization problem can be solved with the GA (goal attainment) method [17]. A sophisticated optimization algorithm applying such a method (referred to as the GA algorithm) has been attempted in our simulation. Since the algorithm was designed for general applications, it disregards the specific properties of the problem and does not even converge under specific circumstances. For a system with reasonably large $K$ and $M$, the computational time needed and the uncertainty of convergence makes the GA algorithm difficult to implement in the FHPG in real time. 


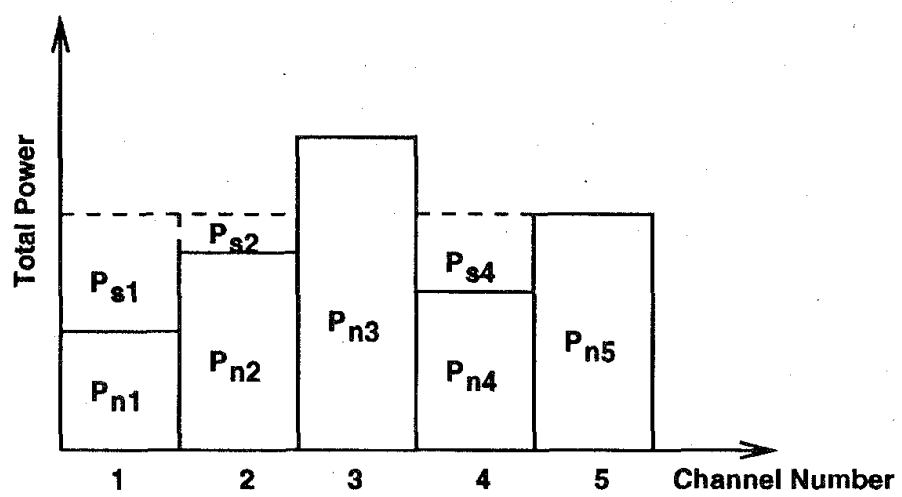

Fig. 2. Water-filling of signal power into parallel channels with Gaussian noise. Pni and Psi stand for the noise and signal power on the ith channel, respectively.

\section{B. Algorithm Design}

In this section, an alternative approach is taken toward solving the optimization problem. Instead of trying to achieve a low BEP directly, we aim at maximizing the average SINR of the system. Although a high SINR is equivalent to low BEP, its expression provides us with more insight into the system behavior.

Equation (13) is rewritten as

$$
\begin{aligned}
\lambda_{k, m} & {\left[\frac{N_{0}}{v_{k, l_{k, m}}}+\frac{\bar{T}_{d}^{2}}{N v_{k, l_{k, m}}}\right.} \\
& \left.\times\left(\sum_{\substack{i=1 \\
i \neq k}}^{K} n_{i, l_{k, m}} v_{i, l_{k, m}}+\left(n_{k, l_{k, m}}-1\right) v_{k, l_{k, m}}\right)\right]^{-1} \\
= & {\left[\operatorname{snr}_{k, m}^{-1}+\operatorname{sir}_{k, m}^{-1}\right]^{-1} }
\end{aligned}
$$

where $\operatorname{snr}_{k, m}$ and $\operatorname{sir}_{k, m}$ are the signal-to-noise ratio (SNR) and signal-to-interference ratio (SIR). $\operatorname{snr}_{k, m}^{-1}$ is defined as the normalized (by received bit energy) noise term (NNT), and $\operatorname{sir}_{k, m}^{-1}$ is the normalized interference term (NIT).

It is noticed that while a large $v_{k, m}$ reduces $\operatorname{snr}_{k, m}^{-1}$, it increases $\operatorname{sir}_{i, m}^{-1}, \quad i \neq k$. Our task is to find a suitable $H$ that produces the smallest possible NNT's and NIT's over the subchannels in use.

A simple recursive algorithm is designed as follows:

Step 0) Define matrix $S$ and initialize matrices $H$ and $S$ : $n_{i, j}=0, s_{i, j}=N_{0} / v_{i, j}, i=1,2, \cdots, K, j=$ $1,2, \cdots, M$.

Step 0.1) Define a set $Q=\{1,2, \cdots, K\}$.

Step 1) Find the $(k, m)$ subchannel such that: $s_{k, m}=\max \left(S_{\min }\right)$, where $S_{\min }=\left\{s_{\min (i)} \mid\right.$ $i \in q\}$, and $s_{\min (i)}=\min \left(s_{i, 1}, s_{i, 2}, \cdots, s_{i, M}\right)$.

Step 2) Assign one substream from the $k$ th user to the $(k, m)$ subchannel and update $S$ as follows: $n_{k, m}=n_{k, m}+1$, and $s_{i, m}=s_{i, m}+\delta_{i, m \mid k}, i=$ $1,2, \cdots, K$, where $\delta_{i, m \mid k}$ is the NIT contributed to subchannel $(i, m)$ by adding one substream to subchannel $(k, m)$

$$
\delta_{i, m \mid k}=\frac{v_{k, m} \bar{T}_{d}^{2}}{v_{i, m} N}
$$

Step 3) Delete the element $k$ from the set $Q$.

Step 4) If $Q \neq \emptyset$, go to Step 1 .

Step 5) Repeat Steps $0.1-4, M$ times.

The design of this algorithm was motivated by the waterfilling (WF) principle in information theory [18], which is illustrated in Fig. 2. This principle states that given parallel channels with Gaussian noise, information should be first fed into channels with lower noise levels to achieve the maximum channel capacity. What prevents its direct application to our problem is the interaction between users through MAI. But the approximation of MAI with a Gaussian distribution has allowed us to treat it as part of the channel noise, and thus to apply the WF principle recursively. In what follows, we refer to the above algorithm as the WF algorithm.

It can be seen that although every user has an opportunity to access its "favorite" subchannel during each iteration, priority is given to the user whose "favorite" subchannel has the lowest signal level among the set of users. The WF algorithm is simple to implement and guaranteed to converge after $K \times M$ iterations. As shown in the next section, the WF algorithm provides a performance nearly as optimal as the GA optimization algorithm, but with a much faster speed. One pitfall might lie in the case where one user's fading amplitudes are much larger than the average. Whereas the GA algorithm may choose to discard some of those especially strong subchannels from interference-consideration and still keep a lower than average BEP for this user, the WF algorithm overlooks such a situation. However, since fading amplitudes are weakly correlated and have equal average bit energies, such a situation occurs rarely.

\section{RESULTS AND DISCUSSION}

The system performance is evaluated using a Monte-Carlo simulation. We assume a system with $1.25 \mathrm{MHz}$ bandwidth. We assume that the user data rate is $R=8 \mathrm{~Kb} / \mathrm{s}$. We set $M=8$, corresponding to a delay spread of about $T_{d}=6 \mu \mathrm{s}$, which is common in urban areas [19]. We assume a processing gain equal to 156 . For a mobile with a velocity between 3 


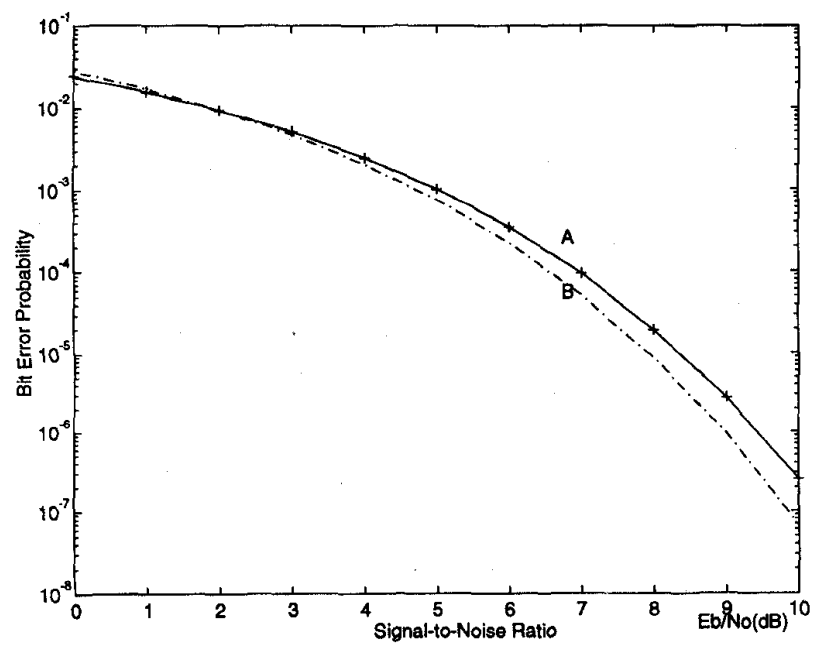

Fig. 3. Average system BEP's obtained with the GA algorithm and with the WF algorithm. $L_{p}=3, T_{d}=6 \mu \mathrm{s}, \kappa=5, M=8$, and $N=156$. A-WF algorithm and $\mathrm{B}-\mathrm{GA}$ algorithm.

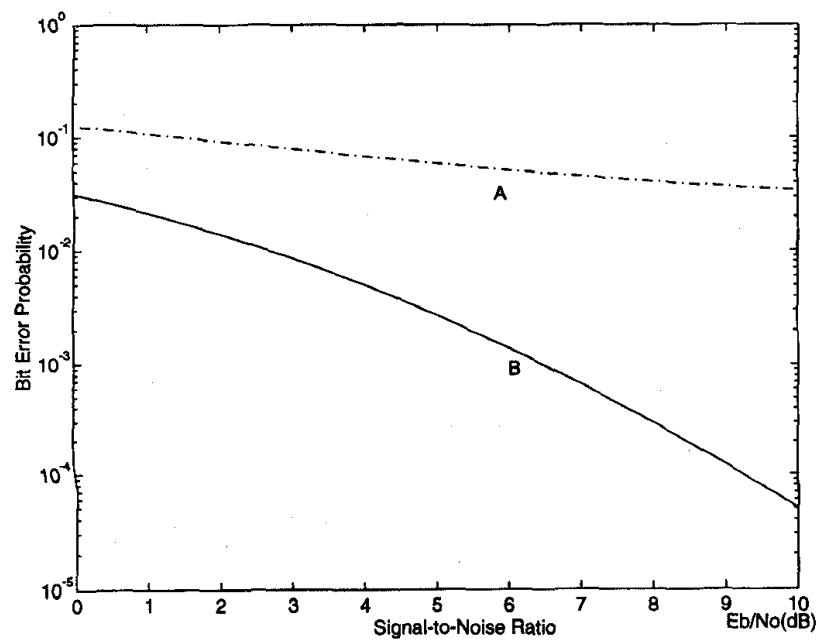

Fig. 4. Average system BEP's obtained with the adaptive frequency hopping MC DS-CDMA system and a SC-DS-CDMA system utilizing a RAKE recciver. $L_{p}=3, T_{d}=6 \mu \mathrm{s}, K=64, M=8$, and $N=156$. A-RAKE system and $\mathrm{B}-$ adaptive MC FH/DS system.

$\mathrm{Km} / \mathrm{H}$ and $50 \mathrm{Km} / \mathrm{H}$, the coherence time ranges from $0.02 \mathrm{~s}$ to $0.4 \mathrm{~s}$, based on which $T_{f h}$ is chosen. Since the data rate is $1 \mathrm{~Kb} / \mathrm{s}$ over each subchannel, at least $24 \sim 400$ data symbols can be transmitted during each hop. The number of multiple paths is set to three in the simulation.

Fig. 3 shows the BEP's (averaged among all users) obtained with the GA algorithm and the WF algorithm. It is seen that the performance obtained with the WF algorithm is slightly inferior. However, even with $K=5$, which is unreasonably small, the time taken by the GA algorithm to reach the solution is almost a thousand times longer than that of the WF algorithm. Hence, the WF algorithm is applied hereafter to evaluate the system performance. $K$ is set to 64 for the rest of the results.

In Fig, 4, the performance of the adaptive MC FH/DS system is compared with a SC-DS-CDMA system using a

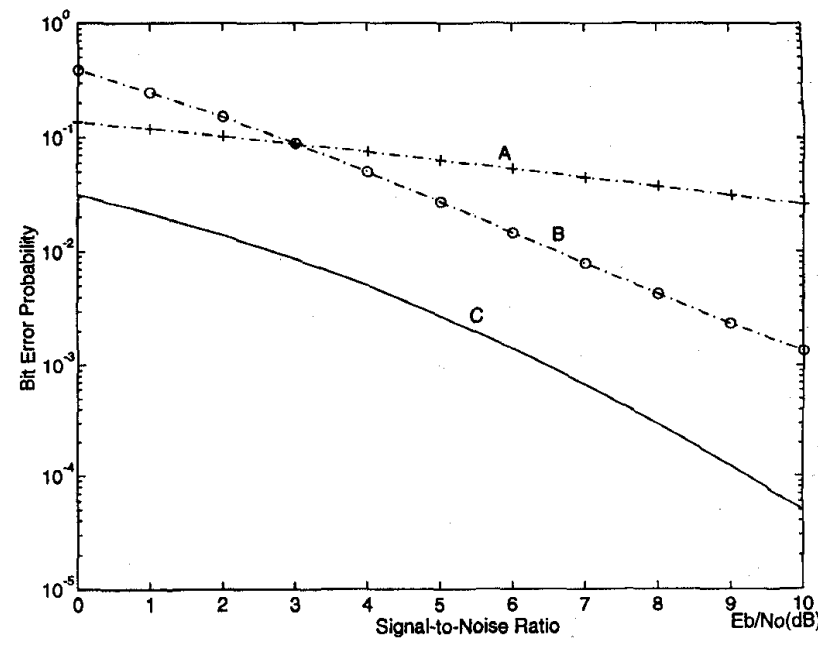

Fig. 5. Average system BEP's obtained with the adaptive frequency hopping MC DS-CDMA system and diversity system using selection combining and maximal ratio combining techniques. $L_{p}=3, T_{d}=6 \mu \mathrm{s}, K^{\circ}=64, M=8$, and $N=156$. A-Diversity system with selection combining, B-diversity system with MRC, and C-adaptive MC FH/DS system.

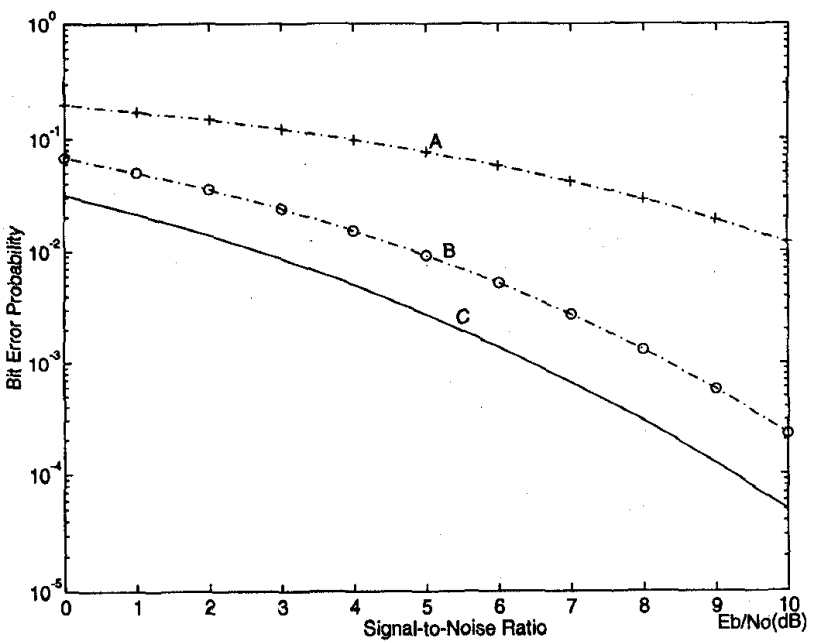

Fig. 6. Average system BEP's obtained with the adaptive frequency hopping $M C$ DS-CDMA system and diversity system using selection combining and maximal ratio combining techniques. $L_{u}=3, T_{d}=6 \mu \mathrm{s}, K=64, M=8$. and $N=156$. A-Diversity system with selection combining, B-diversity system with MRC, and C-adaptive MC FH/DS system.

RAKE receiver. The proposed system performs much better than the single carrier system. There are at least two reasons for this behavior. First, by achieving quasisynchronization, our system effectively reduces the MAI which is a major performance constraint in an asynchronous SC-DS-CDMA system with a large number of users. Second, instead of combining all paths as the RAKE receiver does, with the MC scheme we are effectively taking full advantage of the strongest path.

In Fig. 5, the system performance is compared with the conventional MC CDMA system. Even with the use of a $(32,6)$ Reed-Muller code, the conventional system's BEP performance is still poorer. Though higher complexity is 
the price we pay for the improved performance in the MC scheme, such a complexity should be quite affordable in a CDMA network where even more complicated power control algorithms have been proposed. The frequency hop control scheme may even be combined with a power control scheme.

In Fig. 6, we also compare the performance of the proposed scheme with schemes based on frequency diversity which are frequently used in multipath fading channels. Besides the performance disadvantages, a coded or diversity system may have the drawbacks of bandwidth expansion or data rate reduction.

Aside from the average system BEP, we are also interested in how the substreams are distributed among subchannels. It is found that when AWGN dominates over MAI, substreams tend to concentrate on a few "favorite" subchannels leaving most of the others unused. The opposite happens when MAI dominates over thermal noise. In either case, the total number of substreams hopping to one particular subchannel remains almost equal to $K$ on the average.

\section{CONCLUSION}

A MC DS-CDMA system with adaptive frequency hopping is proposed in this paper. The problem of obtaining the optimal frequency hopping pattern was formulated as a multiobjective optimization problem. An efficient algorithm, based on the WF principle, that could be implemented in practice, has been designed. The system has shown significant performance improvement over some existing systems in terms of the average system BEP. Such an improvement can be directly translated into an increase in CDMA system capacity.

\section{REFERENCES}

[1] N. Yee, J. P. Linnartz, and G. Fettweis, "Multicarrier CDMA in indoor wireless radio networks," in Proc. PIMRC'93, Sept. 1993, pp. 109-113.

[2] K. Fazel and L. Papke, "On the performance of convolutionally-coded CDMA/OFDM for mobile communication system," in Proc. PIMRC'93, Sept. 1993, pp. 468-472.

[3] A. Chouly, A. Brajal, and S. Jourdan, "Orthogonal multicarrier techniques applied to direct sequence spread spectrum CDMA system," in Proc. GLOBECOM'93, Nov. 1993, pp. 1723-1728

[4] N. Yee and J. P. Linnartz, "Wiener filtering of multicarrier CDMA in a Rayleigh fading channel," in Proc. PIMRC'94, Sept. 1994, pp. $1344-1347$.

[5] S. Kondo and L. B. Milstein, "On the use of multicarrier direct sequence spread spectrum systems," in Proc. MILCOM'93, Oct. 1993, pp. 52-56.

[6] _ "Multicarrier DS CDMA system with cochannel interference cancellation," in Proc. VTC'94, June 1994, pp. 1640-1644.

[7] "Multicarrier DS CDMA system in the presence of partial band interference," in Proc. MILCOM'94, Oct. 1994, pp. 588-592.

[8] O. van de Weil and L. Vandendorpe, "Analysis of residual interference after DFE equalization of multitone DS/SS system over multipath channels," in Proc. VTC'95, July 1995, pp. 394-398.

[9] V. DaSilva and E. Sousa, "Performance of orthogonal CDMA codes for quasisynchronous communication systems," in Proc. ICUPC'93, Oct. 1993, vol. 2, pp. 995-999.

[10] Q. Chen, E. Sousa, and S. Pasupathy, "Performance of coded multicarrier DS-CDMA system in multipath fading channels," Wireless Communications, vol. 2, pp. 167-183, 1995.

[11] R. E. Ziemer and R. L. Peterson, Digital Communications and Spread Spectrum Systems. New York: Macmillan, 1985.

[12] G. L. Turin, "Introduction to antimultipath techniques and their applications to urban digital radio," Proc. IEEE, vol, 68, pp. 328-353, Mar. 1980.

[13] A. P. Clark and S. Hariharan, "Adaptive channel estimator for an HF radio link," IEEE Trans. Commun., yol. 37, pp. 918-926, Sept. 1989.
[14] S. A. Fechtel and H. Meyr, "An investigation of channel estimation and equilization techniques for moderately rapid fading HF-channels," in Proc. ICC'91, June 1991, pp. 768-772.

[15] _ "Optimal parametric feedforward estimation of frequencyselective fading radio channels," IEEE Trans. Commun., vol. 42, pp. 1639-1650, Feb./Mar./Apr. 1994.

[16] E. Sousa, "The effect of clock and carrier frequency offsets on the performance of a direct sequence spread spectrum multiple-access system," IEEE J. Select. Areas Commun., vol. 8, pp. 580-587, May 1990.

[17] A. Grace, Optimization Tool Box for Use with Matlab. MA: Math Works, Nov. 1992.

[18] T. M. Cover and J. A. Thomas, Elements of Information Theory. New York: Wiley, 1991.

[19] E. Sousa, V. M. Jovanovic, and C. Daigneault, "Delay spread measurements for the digital cellular channel in Toronto," IEEE Trans. Veh. Technol., vol. 43, pp. 837-847, Nov. 1994.

Qingxin Chen was born in Quanzhou, Fujian, China, on December 17, 1966. She received the B.E. degree in radio engineering from Southeast University, Nanjing, China, in 1989, and the M.A.Sc, degree in electrical engineering from University of Victoria, Victoria, British Columbia, Canada, in 1992

She is currently working toward her doctorate in the Department of Electrical and Computer Engineering, University of Toronto. Her thesis topic is on multicarrier DS-CDMA systems in multipath fading chamels.

Elvino S. Sousa (S'79-M'80) was born in Gracioca, Azores, on December 28,1956 . He received the B.A.Sc. degree in engineering science, and the M.A.Sc. degree in electrical engineering from the University of Toronto in 1980 and 1982 respectively, and the Ph.D. degree in electrical engineering from the University of Southern California, in 1985.

Since 1986, he has been with the Department of Electrical and Computer Engineering, University of Toronto, where he is presently an Associate Professor. Since 1986, he has been a Natural Sciences and Engineering Research Council of Canada (NSERC) University Research Fellow. He has performed research in spread-spectrum systems since 1983. His current interests are in the areas of spread-spectrum systems, mobile communications, and indoor wireless communications with emphasis on the high level performance of the network and signal processing issues affecting such a performance. At the University of Toronto, he has developed a graduate course in mobile communications and also teaches a graduate course in error control codes. He has given numerous tutorials and short courses in wireless communication systems and spread spectrum at various conferences and institutions in over one dozen countries. He has organized various sessions in wireless communications at major conferences and was the Technical Program Chairman for the Sixth IEEE International Symposium on Personal, Indoor and Mobile Radio Communications, Toronto, September 1995 (PIMRC'95).

Subbarayan Pasupathy (M'73-SM'81-F'91) was born in Madras, Tamilnadu, India, on September 21, 1940. He received the B.E. degree in telecommunications from the University of Madras, Madras, in 1963, the M.Tech. degree in electrical engineering from the Indian Institute of Technology, Madras, in 1966, and the M.Phil. and Ph.D. degrees in engineering and applied science from Yale University, New Haven, Cr, in 1970 and 1972, respectively.

From 1965 to 1967, he was a Research Scholar and Part-Time Lecturer at the Indian Institute of Technology, Madras, and worked as a Teaching Assistant at Yale University, New Haven, CT, from 1968 to 1971. During 1972-1973, he was a Post Doctoral fellow at the University of Toronto, Toronto, Ontario, Canada, working in the area of array processing of sonar signals. In 1973, he joined the faculty of the University of Toronto, and became a Professor of Electrical Engineering in 1983. He was the Associate Chairman of the Electrical Engineering Department from 1979 to 1982. At present, he is the Chairman of the Communications Group. His research interests lie in the areas of communication theory, digital communications, and statistical signal processing. He has been a University of Toronto Coordinator of the Network Engineering Program (a joint offering by Northern Telecom and University of Toronto) since its inception in 1987. From 1982 to 1989, he was an Editor for Data Communications and Modulation for the IEEE TRANSACTIONS ON COMmunications. He has served as a Technical Associate Editor for the IEEE Communications Magazine, from 1979 to 1982, and as an Associate Editor for the Canadian Electrical Engineering Journal, from 1980 to 1983. Since 1984, he has been writing a regular column entitled "Light Traffic" for the IEEE COMMUNICATIONS MAGAZINE.

Dr. Pasupathy is a registered Professional Engineer in the province of Ontario. He was elected as a Fellow of the IEEE in 1991 "for contributions to bandwidth efficient coding and modulation schemes in digital communication." 